\title{
CdSe/CdS dot-in-rods nanocrystals fast blinking dynamics.
}

\author{
M. Manceau ${ }^{1,2}$, S. Vezzoli ${ }^{3}$, Q. Glorieux $^{1}$, E. Giacobino ${ }^{1}$, L. \\ Carbone $^{5}$, M. De Vittorio ${ }^{4,5}$, J.-P. Hermier ${ }^{6}$ and A. Bramati ${ }^{1}$ \\ 1 Laboratoire Kastler Brossel, \\ UPMC-Sorbonne Universités, CNRS, \\ ENS-PSL Research University, Collège de France, \\ 4, place Jussieu Case 74, F-75005 Paris, France \\ 2 Université Paris 13, Sorbonne Paris Cité, \\ Laboratoire de Physique des Lasers, \\ F-93430 Villetaneuse, France \\ 3 The Blackett Laboratory, \\ Department of Physics, Imperial College London, \\ London SW7 2AZ, United Kingdom \\ 4 Istituto Italiano di Tecnologia (IIT) Center for Bio-Molecular Nanotechnologies Via Barsanti sn, \\ 73010 Arnesano (Lecce), Italy \\ 5 CNR NANOTEC- Institute of Nanotechnology c/o Campus Ecotekne, \\ University of Salento, Via Monteroni - 73100 Lecce, Italy \\ 6 Groupe dÉtude de la Matière Condensée, \\ Université de Versailles-Saint-Quentin-en-Yvelines, \\ CNRS UMR 8635, 45, Avenue des Etats-Unis, \\ F-78035 Versailles, France
}

(Dated: March 17, 2022)

\begin{abstract}
The blinking dynamics of colloidal core-shell CdSe/CdS dot-in-rods is studied in detail at the single particle level. Analyzing the autocorrelation function of the fluorescence intensity, we demonstrate that these nanoemitters are characterized by a short value of the mean duration of bright periods (ten to a few hundreds of microseconds). The comparison of the results obtained for samples with different geometries shows that not only the shell thickness is crucial but also the shape of the dotin-rods. Increasing the shell aspect ratio results in shorter bright periods suggesting that surface traps impact the stability of the fluorescence intensity.
\end{abstract}

PACS numbers: 8.67.Bf,42.50.Ar,78.55.Cr,79.20.Fv

\section{INTRODUCTION}

The emission intermittency, commonly called blinking, is characteristics of single nanocrystals emission. Since the first measurements on single CdSe nanocrystals ${ }^{11}$ reporting a switching between an ON state emitting photons and an OFF state completely dark, this phenomenon has been the focus of intense studies because it deeply undermines the possible applications foreseen for these emitters, ranging from bio-imaging, light harvesting to nanophotonics $^{2}$ and quantum optics $\frac{344}{4}$ A complete physical picture of the phenomenon has not been reached yet owing to the complexity of the processes at stake.

Fluorescence blinking has been observed for various types of single nanoscale emitters ${ }^{5}$, including molecular dyes, fluorescent proteins, small nanodiamonds ${ }^{6}$ and colloidal nanocrystals. For complex single emitters such as CdSe nanocrystals, a broad distribution of blinking rates is observed, resulting in periods with a large fluorescence (on-state) and low fluorescence (off-state corresponding to the noise level) spanning from microseconds to hundreds of seconds ${ }^{7 / 8}$. Since the first report of fluorescence blinking in small spherical nanocrystal $\$^{1}$, this behavior has been observed for many morphologies including elongated nanorods and nanowires. In the case of nanocrys- tals, power law distributions ${ }^{7}$ with exponent smaller than 1 were reported for cumulative durations of $\mathrm{ON}$ and $\mathrm{OFF}$ events. These so called Levy distributions, have singular statistical properties: no mean value or standard deviation can be defined. Moreover long blinking periods are very probable as the decay of the distribution is slow. Also, more puzzling phenomena are associated with these distributions, such as statistical aging and non ergodicity ${ }^{8}$.

The last years have seen considerable progresses in reducing the effects of blinking thanks to new chemical synthesis methods $\$ \frac{910}{}$ enabling the growth of CdS thick shells around the CdSe emitting core. It results in a better confinement of the charges inside the nanocrystal and the strong modification of the flickering dynamics. Long low-emitting periods are no more observed. Their duration does not exceed $100 \mathrm{~ms}$. In addition, the emission does not turn completely off and "grey" states have been identified 11 . In that sense some articles mention nonblinking nanocrystals, even if super-Poissonian intensity fluctuations remain 1114 indicating flickering between at least two emission levels.

Beyond the growth of thicker shells, the overall nanocrystals shape (core and shell) can now be controlled. For example, dot-in-rods ${ }^{15}-17$ (DRs) consisting 
of a spherical core embedded in a cylindrical shell have been fabricated as well as nanoplatelets $\$ 18$. The modification of the shape opens new opportunities for the understanding and engineering of nanocrystals optical and spectroscopic properties $\$ \frac{1922}{22}$, in particular in view of pure single photon emission.

In this paper, we show that geometry plays an important role in the blinking dynamics of DRs and we propose a method of analysis for the blinking. We first observe that these DRs are characterized by a fast blinking dynamics. From a methodological point of view, our results indicate that it is usually poorly resolved when binning the signal, even with bin times as short as hundreds of microseconds. Due to the fast blinking dynamics, the common blinking analysis 78 based on binning the photon detection events cannot yield any trustworthy information. In order to overcome this problem we show that a better approach consists in measuring the intensity autocorrelation function. In particular, it provides the average duration of bright periods. We then compare DRs samples with different geometries and demonstrate that, in addition to the shell thickness, the aspect ratio of the DR influences crucially the bright period duration.

\section{FAST BLINKING DYNAMICS}

For our study we used high quality $\mathrm{CdSe} / \mathrm{CdS}$ coreshell DRs synthesized using the seeded growth approach proposed in reference ${ }^{17 / 23}$. In Tab 1 we give the core diameters, shell thicknesses and shell lengths of the various samples under study. The DRs are characterized by a minimum number of CdS monolayers on top of the core which is also given in Tab. In the following DR1 corresponds to the thin shell dot-in-rods sample, while DR2, DR3 and DR4 are thick shell samples. For each sample a dilute toluene solution is drop-cast on a microscope glass coverslip to produce a low density of single DRs (typically 2 DRs per $5 \mu \mathrm{m}^{2}$ area). A single DR can be chosen and excited using a picosecondpulsed laser diode with a small excitation spot of $1 \mu \mathrm{m}^{2}$. The picosecond-pulsed laser operates at a wavelength of $405 \mathrm{~nm}$ and excites the highly absorptive shell[17, with a repetition rate of $2.5 \mathrm{MHz}$. The photoluminescence (PL) is collected using a confocal microscope with a high numerical aperture objective $(100 \times$, N.A.=1.4). A high pass filter (cutoff $570 \mathrm{~nm}$ ) removes the remaining excitation light while leaving the DRs PL which is centered around $600-650 \mathrm{~nm}$ for the various samples. The DRs $\mathrm{PL}$ is then spatially filtered through a pinhole and subsequently recorded using two single-photon avalanche photodiodes in a Hanbury-Brown and Twiss configuration. The signals from the photodiodes were recorded by a Time-Correlated Single Photon Counting data acquisition card enabling for each DR the recording of the PL autocorrelation function. Prior to any measurements we therefore check if the chosen particle is single or not with an antibunching measurement.
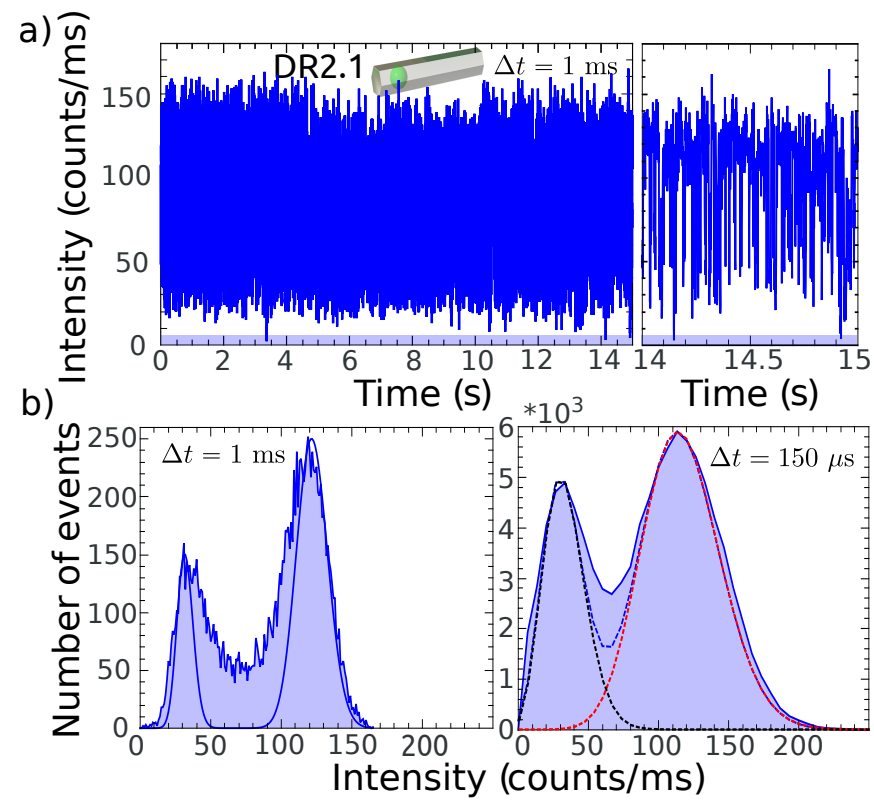

FIG. 1. a) Left: typical PL timetrace of DR2.1 from sample DR2. Right: close view on the last second of the registered timetrace showing the flickering between two states. Mean excitation: $\left\langle N_{e h}\right\rangle=0.5$. Bin time $\Delta t=1 \mathrm{~ms}$. The noise level is given by the faint blue area. b) Histogram of emission corresponding to the PL timetrace in a) for two time bins $\Delta t$. Each histogram is renormalized in counts $/ \mathrm{ms}$. Left: $\Delta t=$ $1 \mathrm{~ms}$, right: $\Delta t=150 \mu \mathrm{s}$. A fit with the sum of two Poisson distributions (dashed blue line) is given in each case.

We present the PL timetrace of a typical dot-in-rod of the thick shell sample DR2 in Fig:11 for an excitation below saturation. In the following In the following we call DR2.1 this particular DR from sample DR2. The average number of electron-hole pairs $\left\langle N_{e h}\right\rangle$ inside the structure was measured to be $\left\langle N_{e h}\right\rangle=0.5$ by a saturation measurement ${ }^{24}$. The bin time is $\Delta t=1 \mathrm{~ms}$. The corresponding histogram of emission is shown in Fig $1 \mathrm{p}$ left. The histogram of emission for a PL timetrace computed with a $\Delta t=150 \mu \mathrm{s}$ bin time is also shown in Fig $1 \mathrm{~b}$ right. This histogram reveals the presence of two emission peaks as expected for these emitter ${ }^{25}$ for an excitation under saturation. Indeed, DRs with a thick shell such as DR2.1 have a reduced blinking between a bright and a grey state, as demonstrated using time resolved decay measurements in one of our previous work ${ }^{25}$, corresponding respectively to the radiative recombination of an exciton and a negative trion ${ }^{11113}$ with a lower emission rate. The low emission state is well above the noise level here (3 count/ms shown in faint blue on Fig 1 a $)$. The intensity of emission of the bright and grey states are $I_{X}=116$ Counts $/ \mathrm{ms}$ and $I_{X^{-}}=33$ Counts $/ \mathrm{ms}$ respectively. The histograms of emission can be fitted with the sum of two Poisson distributions (dashed blue line). In principle each emission state should correspond to a single Poisson distribution once the signal is properly binned. However Fig $1 \mathrm{p}$ clearly shows the limitations of 
such a fitting procedure.

One can see in Fig 1 1 a that the emission is characterized by a fast switching between the two states. Indeed the $\Delta t=1 \mathrm{~ms}$ bin time in Fig, $1 \mathrm{~b}$ left can only poorly resolve the emission dynamics. A broad range of intermediate emission intensities is visible in between the two emission peaks because of the time averaging imposed by the binning of the data. The binning of $\Delta t=150 \mu \mathrm{s}$ is far more accurate in resolving the emission dynamics as a better although, not perfect agreement is found with the two state emission fit. When using a technique of analysis of the signal relying on binning the photon detection events together, it is important to optimize the value of the time bin. The question of finding an "optimized" bin time is intrinsically linked to the timescales at which the blinking process occurs. In the nanocrystals literature, most of the publications present data with bin times of $10 \mathrm{~ms}$ or more. Large time bins are not suitable for the DRs under investigation. However, it is also important to state that the bin time cannot be set to extremely short values. Indeed, one is also limited by the photon collection rate, here $\mathrm{a} \simeq 100$ counts $/ \mathrm{ms}$ for the chosen excitation. The shorter the bin time the fewer detection events per time bin and the broader the corresponding Poisson distribution. Short time bins lead therefore to overlapping distributions as is visible in Fig $1 \mathrm{~b}$ right for $\Delta t=150 \mu$ s where the two Poisson distribution clearly overlap. Ultimately, a bin time of the order of the emitter lifetime will lead to on average less than one photon per time bin, with grey and bright states becoming completely indistinguishable. Indistinguishable grey and bright states due to overlapping distributions are a problem when a threshold needs to be set to distinguish the two states as will be seen in the next section.

\section{CHARACTERIZING THE BLINKING DYNAMICS}

We now present a more quantitative analysis of the reduced blinking dynamics of thick shell DRs. In Fig 2 a and Fig $2 \mathrm{~b}$ we present the cumulative distributions of the bright $\left(\mathcal{P}_{b}\left(\tau_{b} \geq \tau\right)\right)$ and grey $\left(\mathcal{P}_{g}\left(\tau_{g} \geq \tau\right)\right)$ states event durations from the DR2.1 timetrace presented on Fig 1 . The cumulative distribution $\mathcal{P}_{b, g}\left(\tau_{b, g} \geq \tau\right)$ as a function of $\tau$ gives the probability that the bright (grey) period $\tau_{b}\left(\tau_{g}\right)$ is larger than $\tau$. Bin times of $\Delta t=150 \mu \mathrm{s}$ and $\Delta t=1 \mathrm{~ms}$ are used respectively. The thresholds $I_{b}$ and $I_{g}$ for the bright and grey states events are fixed in between the two states at $I_{b}=I_{g}=60$ counts $/ \mathrm{ms}$. For the case of $\Delta t=150 \mu \mathrm{s}$, this threshold value corresponds roughly to a distance of 5 standard deviation to the mean value of each emission state. This way a minimum of overlap between the states is ensured as can be seen from the fit in Fig $1 \mathrm{p}$ right and no data is discarded from the analysis in this case. Time bins with intensities above $I_{b}$ are considered as part of the bright state and time bins with intensities below $I_{g}$ are considered as part of the grey state. The cumulative distributions shows that long periods are strongly inhibited for both type of events, grey or bright as expected from the timetrace in Fig.17. Fits of the cumulative distributions are presented in Fig, $2 \mathrm{a}$ as full lines corresponding to power laws with exponential cutoff:

$$
\mathcal{P}\left(\tau_{b, g} \geq \tau\right) \propto \frac{1}{\tau^{\mu}} e^{-\tau / \tau_{c}},
$$

with $\mu$ the power law exponent and $\tau_{c}$ the exponential cutoff time. For $\Delta t=150 \mu \mathrm{s}$, we obtain power law exponents of 0.46 and 0.42 for the grey and bright states respectively with exponential cutoffs of 1.5 and $6.1 \mathrm{~ms}$ for the cumulative distributions. Here we report values of the power law exponent $\mu$ corresponding to cumulative distributions. Hence the values close to $0.5^{8}$ corresponds to $1+\mu \simeq 1.5$ often reported in the literature for the non-cumulative distributions.

However it should be noted that in the case of two states with close emission rates, the analysis of event durations is not totally reliable, indeed it strongly depends on the thresholds chosen and also on the bin time $\Delta t$ of the intensity timetrace as demonstrated in reference $26 \mid 27$. In Fig. $2 \mathrm{~b}$ we present the same analysis with a larger bin time than in Fig $2 \mathrm{a}, \Delta t=1 \mathrm{~ms}$, and the same thresholds. The curves have the same shapes, the fits yield power law exponents of 0.24 and 0.16 for the grey and bright states respectively with exponential cutoffs of 3.8 and $10.5 \mathrm{~ms}$. Changing the bin time has considerably modified the distributions and the fitting values. We already know that the bin time of $\Delta t=1 \mathrm{~ms}$ is less relevant that the bin time of $\Delta t=150 \mu$ s for the studied timetrace as explained in the previous section. Many events in the PL timetrace have durations between $150 \mu$ s and $1 \mathrm{~ms}$ as attested by the slope of the cumulative distribution on these timescales in Fig. $2 \mathrm{p}$. Indeed, approximately $63 \%$ of the events considered as bright with the bin time of $\Delta t=150 \mu$ s have durations smaller than $\tau=1 \mathrm{~ms}$ as $\mathcal{P}_{b}\left(\tau_{b} \geq 1 \mathrm{~ms}\right)=37 \%$ in Fig 2 a. Hence all these events cannot be resolved with a bin time of $\Delta t=1 \mathrm{~ms}$. Furthermore, bright events with duration shorter than a $150 \mu$ s also exist, but cannot be grasped by the $\Delta t=150 \mu \mathrm{s}$ binning. This explains the imperfect fitting by the two Poisson distributions in Fig, $1 \mathrm{p}$ right.

In the following we will investigate the blinking between the bright and grey states using a different approach: the intensity correlation function 28,35 . The autocorrelation method is less straightforward than the distribution of event durations, but it does not suffer from any a priori assumptions due to the time bin and it yields information at short timescales not reachable when binning the signal.

Fig 2 c presents the $g^{(2)}$ function corresponding to the PL timetrace in Fig.1. The temporal intensity correlation $g^{(2)}$ of a light field is defined as follows: 


$$
g^{(2)}\left(t_{0}, t_{0}+\tau\right)=\frac{\left\langle I\left(t_{0}\right) I\left(t_{0}+\tau\right)\right\rangle}{\left\langle I\left(t_{0}\right)\right\rangle^{2}},
$$

with $t_{0}, t_{0}+\tau$ times, \langle\rangle denotes a statistical (ensemble) average. For the DRs presented in this paper switching between two emission states characterized by power law distributions with exponential cutoffs of the event durations (eq 1), an expression for the $g^{(2)}$ function is derived in references 3136 . Such a model can be applied to investigate the flickering of $\mathrm{CdSe} / \mathrm{CdS}$ colloidal emitters since the duration of the low emitting periods does not exceed tens of milliseconds 9 , meaning that a cutoff is always observed as can be seen in Fig 2 a. On timescales smaller than the exponential cutoffs of the order of a couple of milliseconds the $g^{(2)}$ function can be expressed as:

$$
g^{(2)}(\tau)=B\left(1-A \tau^{1-\mu}\right)
$$

Here, $\mu$ is the largest power law exponent among the two states power law distributions. $B$ is the bunching value, i.e. the value of $g^{(2)}$ in eq3 at short timescales. Here the model does not take into account antibunching and single photon emission 25 at short timescales of the order of the emitter lifetime (nanoseconds to hundreds of nanoseconds). Therefore, as $\lim _{\tau \rightarrow 0} \tau^{1-\mu}=0, g^{(2)}(0)$ is equal to the bunching value $B$ in eq, 3 . For molecules and quantum dots with simple exponential blinking laws $28|29| 37 \mid 39$, the bunching value corresponds to the ratio between the average $\mathrm{OFF}$ and $\mathrm{ON}$ periods. More generally, the fact that $g^{(2)}$ is larger than one on some timescales means that bunches of photons with various emission rate coexist. This leads to intensity fluctuations larger than Poisson fluctuation corresponding to a single rate of emission and consequently $g^{(2)}>1$. Furthermore, as shown in reference $^{36}$ the parameter $A$ is equal to:

$$
A=\frac{1}{\left\langle\tau_{b}\right\rangle} \frac{\tau_{\min }^{\mu}}{\Gamma(2-\mu)},
$$

with $\left\langle\tau_{b}\right\rangle$ the average duration of the bright state events, $\tau_{\text {min }}$ the minimum duration of a event and $\Gamma$ the usual gamma function. For our experiment, the minimum duration of an emission event $\tau_{\text {min }}$ is equal to the measurement time resolution ${ }^{32}, 400 \mathrm{~ns}$, corresponding to the delay between two excitation pulses. The factor $A$ is inversely proportional to the average duration of the bright state. Hence $\left\langle\tau_{b}\right\rangle$, together with $\mu$, defines on which timescales the $g^{(2)}$ falls towards unity as the blinking correlations are lost due to the cutoffs at long time scales.

In the following, we use the notation $\left\langle\tau_{b}\right\rangle_{d}$ and $\left\langle\tau_{b}\right\rangle_{g^{(2)}}$ for the average bright period duration obtained from the blinking distribution and the correlation function respectively. $\left\langle\tau_{b}\right\rangle_{d}$ is the mean value obtained from the experimental probability distributions, not the cumulative distributions presented in this article. The probability
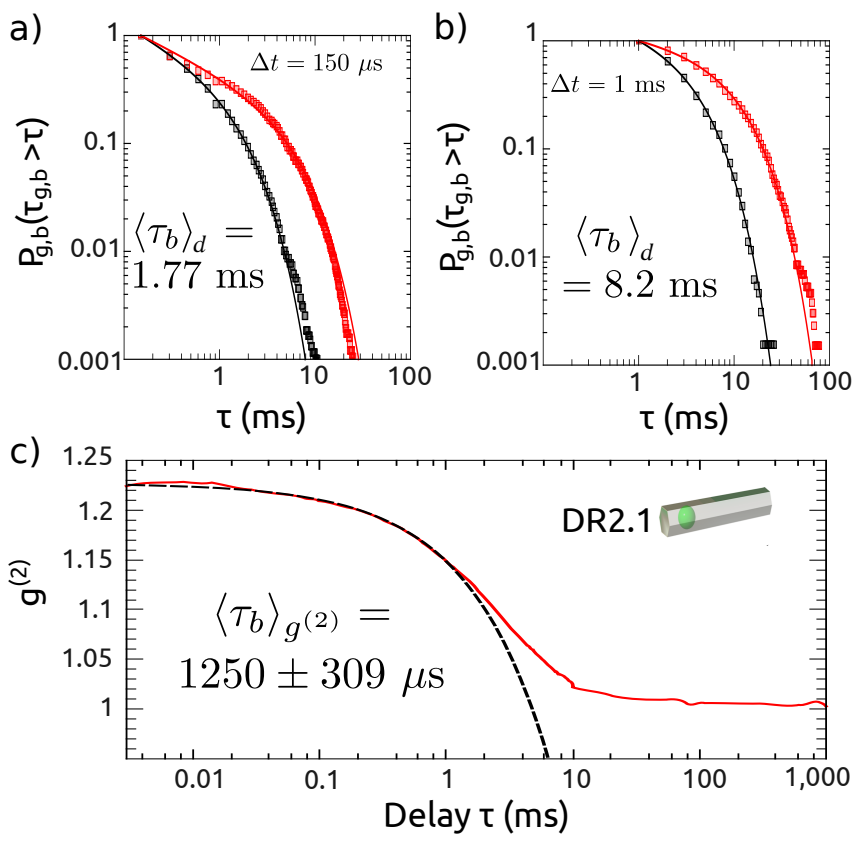

FIG. 2. a) Cumulative distributions of the bright and grey states event durations for DR2.1 corresponding to the histogram presented on Fig 1 b right. Red: bright state, black: grey state. Bin time $\Delta t=150 \mu \mathrm{s}$, thresholds: $I_{b}=I_{g}=$ 60 counts/ms. Full lines: power law distribution with exponential cutoff fit (red: $\mu=0.42$ and $\tau_{c}=6.1 \mathrm{~ms}$, black: $\mu=0.46$ and $\tau_{c}=1.5 \mathrm{~ms}$ ), see Eq1. b) Cumulative distributions of the bright and grey states event durations corresponding to the histogram presented on Fig $1 \mathrm{p}$ left. Red: bright state, black: grey state. Bin time $\Delta t=1 \mathrm{~ms}$, thresholds: $I_{b}=I_{g}=60$ counts $/ \mathrm{ms}$. Full lines: power law distribution with exponential cutoff fit (red: $\mu=0.16$ and $\tau_{c}=10.5 \mathrm{~ms}$, black: $\mu=0.24$ and $\tau_{c}=3.8 \mathrm{~ms}$ ), see Eq 1 c) $g^{(2)}$ function for the detection events presented in Fig 1 . Black dashed line: fit using Eq 3 . This gives $\left\langle\tau_{b}\right\rangle_{g^{(2)}}=1250 \pm 309 \mu \mathrm{s}$ and $\mu=0.34 \pm 0.036$.

distribution of bright events for DR2.1 corresponding to the cumulative distribution presented in Fig 2 yields $\left\langle\tau_{b}\right\rangle_{d}=1.77 \mathrm{~ms}$ with $I_{b}=60$ counts $/ \mathrm{ms}$ and $\Delta t=150 \mu \mathrm{s}$. In order to determine $\left\langle\tau_{b}\right\rangle_{g^{(2)}}$ we fitted the $g^{(2)}$ curve in Fig 2 by Eq, 3 and 4 (see fitting method in Appendix Afor more details). We find $\left\langle\tau_{b}\right\rangle_{g^{(2)}}=1250 \pm 309 \mu \mathrm{s}$, the $309 \mu$ s uncertainty being the fitting error (see Appendix A. The value is smaller than the one found with the blinking distribution. This can be explained by the fact that the blinking distribution does not take into account the fast events (faster than the bin time of $\Delta t=150 \mu \mathrm{s}$ ). Hence and as already stated in reference ${ }^{7}$, the average times deduced from the blinking distributions depend on the bin time chosen because of the scale invariance of the power law. It is interesting to state that the result of a mixing between the two states due to a poor bin or threshold choice is a longer average time, even though grey periods are on average shorter than bright periods (the grey periods distribution is under the bright periods distribution in Fig 2 a). For example $\left\langle\tau_{b}\right\rangle_{d}=1.77 \mathrm{~ms}$ 
was obtained with the threshold $I_{b}=60$ counts $/ \mathrm{ms}$ and $\Delta t=150 \mu \mathrm{s}$. For the case of a bin time $\Delta t=1 \mathrm{~ms}$ in Fig $2 \mathrm{~b}$, the distribution of bright period gives a very poor estimation of the average bright period: $\left\langle\tau_{b}\right\rangle_{d}=8.2 \mathrm{~ms}$. This can be easily understood as the mixing between the two states tends to create long periods with the same intensities. Ultimately, a very large bin would give an average event duration of the order of the measurement time.

For the specific nanocrystal DR2.1 of Fig 1 and Fig 2 , an appropriate choice of bin time can thus fairly well resolve the blinking dynamics as the average switching time between the two states is large enough. A rough estimation of the blinking dynamics can be made using the distribution of bright and grey blinking periods although the results is still biased from the choice of a threshold. In the case of the DR2.1, one should also note that $\left\langle\tau_{b}\right\rangle_{g^{(2)}}=1250 \mu \mathrm{s}$ is close to the time scale for which the fit provided by eq. 4 is valid. This reduces the accuracy of the $\left\langle\tau_{b}\right\rangle_{g^{(2)}}$ value.

In the next section we show some examples for which the use of the distributions of bright and grey blinking periods is irrelevant. The autocorrelation function is then necessary. Various DR samples are studied and we demonstrate that the nanocrystals geometry has an impact on the blinking dynamics.

\section{BLINKING DYNAMICS AND NANOCRYSTAL GEOMETRY}

Fig 3 presents the histograms of emission and $g^{(2)}$ functions for DR3.1, DR4.1, and DR1.1, three representatives DRs of respectively samples DR3, DR4, and DR1, for approximately the same mean excitation $\left\langle N_{e h}\right\rangle=0.5$ as in the former case of DR2.1. One can see that the blinking dynamics of DR3.1 should be characterized by short bright and grey periods. Indeed the histogram of emission in Fig 3 a left has a broad intermediate intensity range in between the two emission peaks due to the bin averaging even though a short bin time of $\Delta t=150 \mu \mathrm{s}$ is used. The analysis in terms of cumulative distributions of blinking periods is inappropriate in this case. The fast blinking dynamics can nevertheless be quantitatively estimated by the fit of the $g^{(2)}$ function in Fig 3 a right. It yields an average bright period duration twenty times shorter than for DR2.1 (Fig 1 and Fig, 2) with $\left\langle\tau_{b}\right\rangle_{g^{(2)}}=42 \pm 8 \mu \mathrm{s}$.

The $g^{(2)}$ curve for DR4.1 appears to be almost flat for $\tau<500 \mu$ s in Fig 3 p right as for DR2.1 in Fig.2k. The average bright periods duration is long compared to DR3.1, $\left\langle\tau_{b}\right\rangle_{g^{(2)}}=465 \pm 122 \mu \mathrm{s}$ is found through the fit of the intensity correlation function. The switching dynamics is therefore on average slower than for DR3.1. The intensity histogram with a $\Delta t=150 \mu$ s bin time is well represented by the sum of two Poisson distributions in Fig $3 \mathrm{~b}$ left. It is to be noted that the two states of emission overlap on a larger intensity range than for the previous exam-
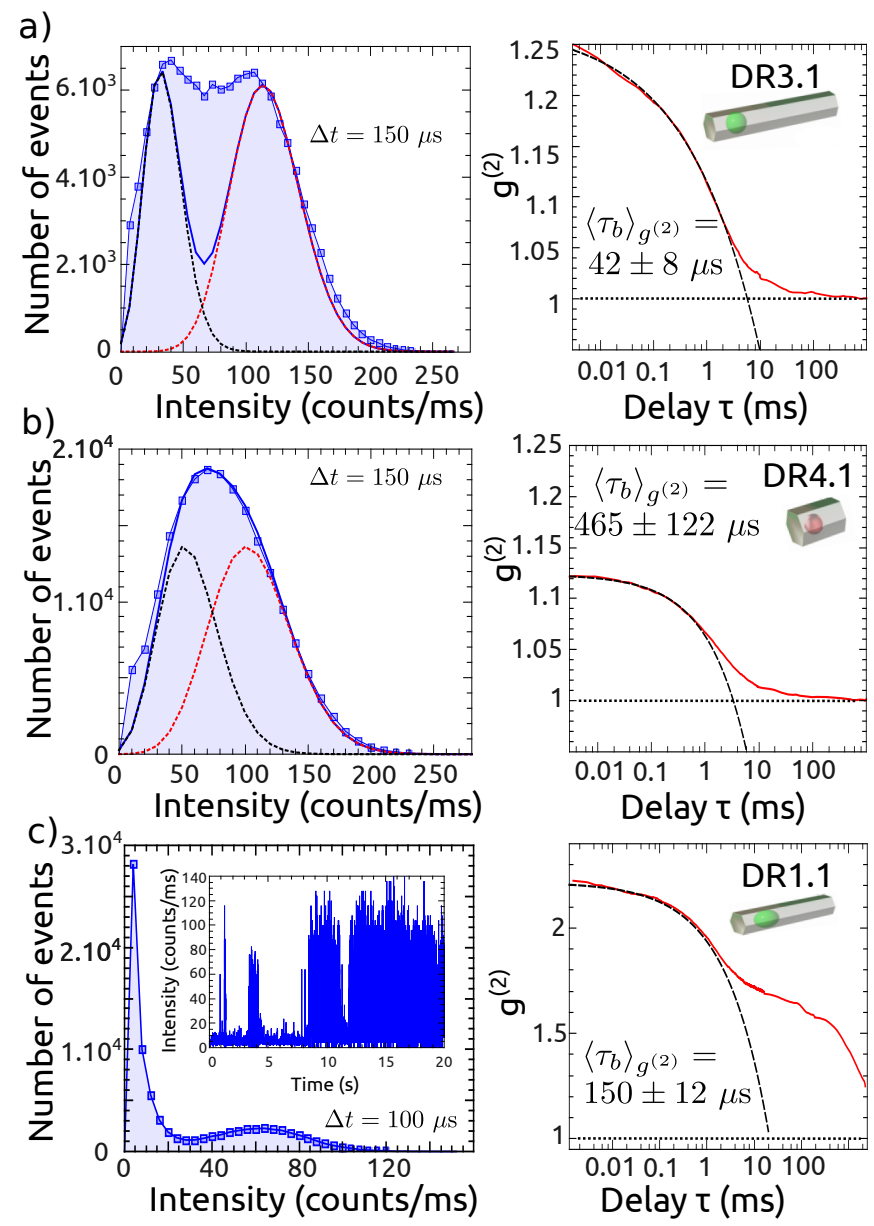

FIG. 3. Histograms and $g^{(2)}$ function for DR3.1, DR4.1 and DR1.1 from samples DR3, DR4 and DR1 respectively, excited at $\left\langle N_{e h}\right\rangle \leq 0.5$. Right panels, red curve: $g^{(2)}$ function, black dashed curve: fit with eq3, a) DR3.1, bin time $\Delta t=150 \mu \mathrm{s}$, $\left\langle\tau_{b}\right\rangle_{g^{(2)}}=42 \pm 8 \mu \mathrm{s}$, and $\mu=0.69 \pm 0.025$. b) DR4.1, bin time $\Delta t=150 \mu \mathrm{s},\left\langle\tau_{b}\right\rangle_{g^{(2)}}=465 \pm 122 \mu \mathrm{s}$, and $\mu=0.49 \pm 0.034$. c) DR1.1, bin time $\Delta t=100 \mu \mathrm{s},\left\langle\tau_{b}\right\rangle_{g^{(2)}}=150 \pm 12 \mu \mathrm{s}$ and $\mu=0.52 \pm 0.01$.

ples. DR4.1 has a very thick shell and therefore a larger trion quantum yield ${ }^{12 \mid 14}$. In this case the estimation of the blinking statistics with the cumulative distributions is inappropriate ${ }^{26}$ as the two states distributions largely overlap and the grey and bright photons are mixed when binning the signal.

We also present the results for thinner shell dot-inrods DR1.1 from sample DR1. In contrast to the previous thick shells samples, this sample switches between a bright and a dark (noise level) state rather than grey state. Also it sometimes shows long dark periods of some seconds, while the bright periods are as previously always limited to a maximum of a few hundreds of microseconds. This is clearly visible in Fig 3 r left that shows the PL (inset) and the corresponding intensity histogram of such a DR with a bin time of $\Delta t=100 \mu \mathrm{s}$. The corresponding $g^{(2)}$ has characteristics similar to the other DRs corre- 
lation functions at short timescales $(<10 \mathrm{~ms})$. This is due to the part of the PL timetrace measured after $10 \mathrm{~s}$ of PL recording for which a fast switching dynamics is observed in Fig.3. left inset. The intensity correlation function nevertheless displays an additional bunching tail at larger timescales $(\tau>10 \mathrm{~ms})$ due to the long blinking events characteristics of thin shell nanocrystals. These long blinking events are visible in the first 10 seconds of the PL recording. The additional decreasing tail of the intensity correlation function for $\tau>500 \mathrm{~ms}$ is due to the finite acquisition time. The fit of the $g^{(2)}$ curve in Fig.35 right gives $\left\langle\tau_{b}\right\rangle_{g^{(2)}}=150 \pm 12 \mu \mathrm{s}$. Let us also note that for these DRs showing fast blinking, the value of $\left\langle\tau_{b}\right\rangle_{g^{(2)}}$ is also very accurate since the agreement between the experimental results and the fit is good for time scales much higher than $\left\langle\tau_{b}\right\rangle_{g^{(2)}}$.

Finally, we present an analysis of the PL of more than 40 DRs for each sample presented previously. The excitation was kept below saturation, in between $\left\langle N_{e h}\right\rangle=0.1$ and $\left\langle N_{e h}\right\rangle=0.5$ for each DR to avoid the excitation of higher order states and to stay in the two states blinking regime $13114 \mid 25$. The intensity correlation function was computed for each DR and fitted with $\mathrm{Eq} 3$ following the procedure described in App $\mathrm{A}$. The sample mean values of the average bright period duration is reported for each sample in Tab. The samples mean values are within a range of 180 to $400 \mu \mathrm{s}$. Our DRs are therefore characterized by a fast switching dynamics that can be only poorly resolved when binning the signal on hundreds microseconds to milliseconds as previously stated. A large dispersion of values exists within each sample, with some DRs having average bright periods of a few tenth of microseconds but also up to $1 \mathrm{~ms}$ as presented in Fig.2. This might be due to a slight dispersion in sizes within a given sample and different electrostatic environment between single dots. The histograms of the various values of bright periods found for each sample are given in App B. It is apparent on these histograms that samples DR1 and DR3 have more single DRs with very short average bright periods (shorter than $100 \mu \mathrm{s}$ ) than samples DR2 and DR4.

We can also notice that even though samples DR1, DR2 and DR3 have the same core, they have different average bright time durations. As expected, the comparison between samples DR1 and DR2 shows that an increase of the shell thickness results in a decrease of the switching dynamics from the bright state to the grey one. However, the DR shell aspect ratio, i.e. the length of the shell over its thickness, is also crucial. Even if samples DR2 and DR3 exhibit the same core diameter and shell thickness, Table I indicates that the increase of the DR length deteriorates the bright state stability. Surface trap states on the shell may be responsible of this observation. A larger shell aspect ratio leads to a faster blinking dynamics. Sample DR3, with a shell aspect ratio of 8.3, has an average bright period duration 1.6 times shorter than sample DR2 with a shell aspect ratio of 3.1. The results concerning sample DR4 confirm the previous obser- vations pointing towards surface trap states as responsible for the instability of the bright state rather than traps inside the nanocrystals volume. Indeed, the synthesis of a very thick shell enables to significantly increase $\left\langle\tau_{b}\right\rangle$ beyond the value obtained for sample DR2 that exhibits approximately the same aspect ratio as sample DR4.

\section{CONCLUSION}

In conclusion, an analysis of the fast blinking dynamics of single colloidal CdSe/CdS DRs was presented. This analysis relies on the autocorrelation function which is shown to be the most suitable tool to properly characterize the flickering between emission states. We showed that the average duration of the bright periods of such emitters is of the order of few hundreds of microseconds, it is so short that any method relying on binning the signal can not resolve appropriately the flickering dynamics while the autocorrelation function gives access to the full blinking dynamics. We also characterized the blinking of several DR samples. Our results demonstrate that not only the thickness of the shell but also its shape has to be considered. The decrease of the bright period duration with the aspect ratio of the shell suggests that traps at the surface of the DR are involved in the flickering of the emission.

\section{Appendix A: fitting of the correlation function}

The normalized correlation functions $g^{(2)}$ were fitted by Eq3.

$$
g^{(2)}(\tau)=B\left(1-A \tau^{1-\mu}\right),
$$

with $B, A$ and $\mu$ as free fitting parameters. $\left\langle\tau_{b}\right\rangle_{g^{(2)}}$ is subsequently determined for each nanocrystal following $\mathrm{Eq} 4$.

$$
A=\frac{1}{\left\langle\tau_{b}\right\rangle} \frac{\tau_{\min }^{\mu}}{\Gamma(2-\mu)},
$$

with $\tau_{\min }=400 \mathrm{~ns}$ the measurement time resolution. One unknown parameter is the long time exponential cutoff as defined in Eq1 1 that characterizes the distribution of bright and grey periods. Eq 3 is valid for correlation times shorter than the exponential cutof ${ }^{3136}$. A limit $\tau_{f}$ to the fit has to be chosen for each DR.

Fig $4 \mathrm{~b}$ presents the normalized residuals for the $g^{(2)}$ fits presented in fig/2 and fig 3 against the fit limit $\tau_{f}$. The residual was normalized to its maximum value at $\tau_{f}=10 \mathrm{~ms}$. For $\tau_{f}>10 \mathrm{~ms}$ the residual increases and the fit becomes clearly inappropriate.

We can see that the residual stays almost constant up to roughly $\tau_{f}=2 \mathrm{~ms}$ before increasing for larger cutoff as the model cannot fit the $g^{(2)}$ curves for larger delays. The 


\begin{tabular}{|l|c|c|c|c|c|c|}
\hline & $\begin{array}{c}\text { Core diameter } \\
(\mathrm{nm})\end{array}$ & $\begin{array}{c}\text { Thickness } \\
(\mathrm{nm})\end{array}$ & $\begin{array}{c}\text { Length } \\
(\mathrm{nm})\end{array}$ & Aspect ratio & $\begin{array}{c}\text { CdS monolayers } \\
\text { on top of CdSe core }\end{array}$ & $\left\langle\tau_{b}\right\rangle_{g^{(2)}}(\sigma)$ \\
\hline \hline DR1 & 3.3 & 4 & 22 & 5.5 & 1 & $190 \mu \mathrm{s}(189)$ \\
\hline DR2 & 3.3 & 7 & 22 & 3.1 & 4 & $299 \mu \mathrm{s}(264)$ \\
\hline DR3 & 3.3 & 7 & 58 & 8.3 & 4 & $186 \mu \mathrm{s}(175)$ \\
\hline DR4 & 4.6 & 11 & 29 & 2.6 & 8 & $394 \mu \mathrm{s}(299)$ \\
\hline
\end{tabular}

TABLE I. Geometrical parameters of the investigated samples. The aspect ratio is the ratio between the length and the thickness of the shell. The last column gives the samples mean values and dispersions for the bright state average duration obtained from the autocorrelation function of more than 40 DRs per sample. See Fig 5 in App B for the various samples $\left\langle\tau_{b}\right\rangle_{g^{(2)}}$ distributions.

corresponding $\left\langle\tau_{b}\right\rangle_{g^{(2)}}$ values against the fit limit $\tau_{f}$ are shown in Fig 4 . The different curves shows that $\left\langle\tau_{b}\right\rangle_{g^{(2)}}$ is almost constant for $\tau_{f}<2$ ms before abruptly dropping while the fit residuals increase for $\tau_{f}>2 \mathrm{~ms}$. The $\left\langle\tau_{b}\right\rangle_{g^{(2)}}$ values reported for single nanocrystals in this article are the average values of the fit for $\tau_{f}<2 \mathrm{~ms}$, while the given fitting errors correspond to the standard deviation of the various fitted values over the range $\tau_{f}<2 \mathrm{~ms}$.
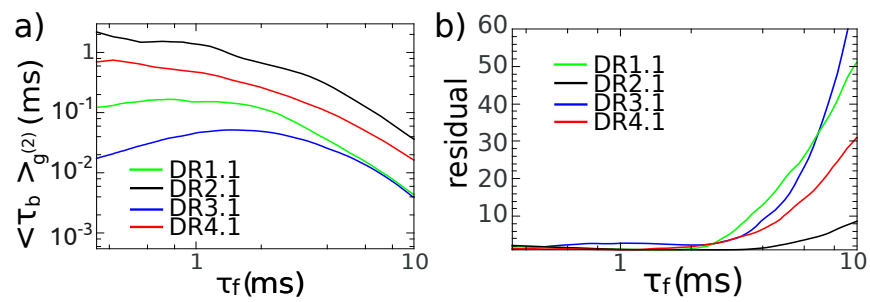

FIG. 4. a) $\left\langle\tau_{b}\right\rangle_{g^{(2)}}$ against the fit limit $\tau_{f}$ for the $g^{(2)}$ curves presented in fig 2 and fig 3 Averaging the fitted values for $\tau_{f}<2 \mathrm{~ms}$ for each DR gives: DR1.1 (green) $\left\langle\tau_{b}\right\rangle_{g^{(2)}}=150 \pm$ $12 \mu \mathrm{s}, \mathrm{DR} 2.1$ (black) $\left\langle\tau_{b}\right\rangle_{g^{(2)}}=1250 \pm 252 \mu \mathrm{s}$, DR3.1 (blue) $\left\langle\tau_{b}\right\rangle_{g^{(2)}}=42 \pm 8 \mu \mathrm{s}$ and DR4.1 (red) $\left\langle\tau_{b}\right\rangle_{g^{(2)}}=465 \pm 122 \mu \mathrm{s}$ b) Fit normalized residual against fit threshold $\tau_{f}$.

\section{Appendix B: Sample distributions of $\left\langle\tau_{\mathbf{b}}\right\rangle_{\mathbf{g}^{(2)}}$}

Following the fitting procedure explained in Appendix A for each single nanocrystals, the sample distributions of average bright periods duration shown in Fig 5 were found by measuring the intensity correlation function of more than 40 single nanocrystals per sample. The samples mean values and standard deviations reported in

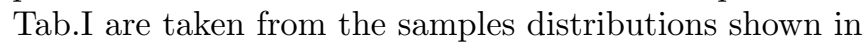
Fig 5 .

\section{Appendix C: Excitation rate and $\tau_{\min }$}

The parameter $\tau_{\text {min }}$ in eq, 4 is the minimum duration of a bright or grey event. In reference ${ }^{36}$, it is included in the model as the short timescale cutoff of the blinking duration distributions $\mathcal{P}\left(\tau_{b, g} \geq \tau\right) \propto \frac{1}{\tau^{\mu}} e^{-\tau / \tau_{c}}$. These distributions need a short time cutoff because they diverge for $\tau \rightarrow 0$. Physically the blinking cannot be infinitely
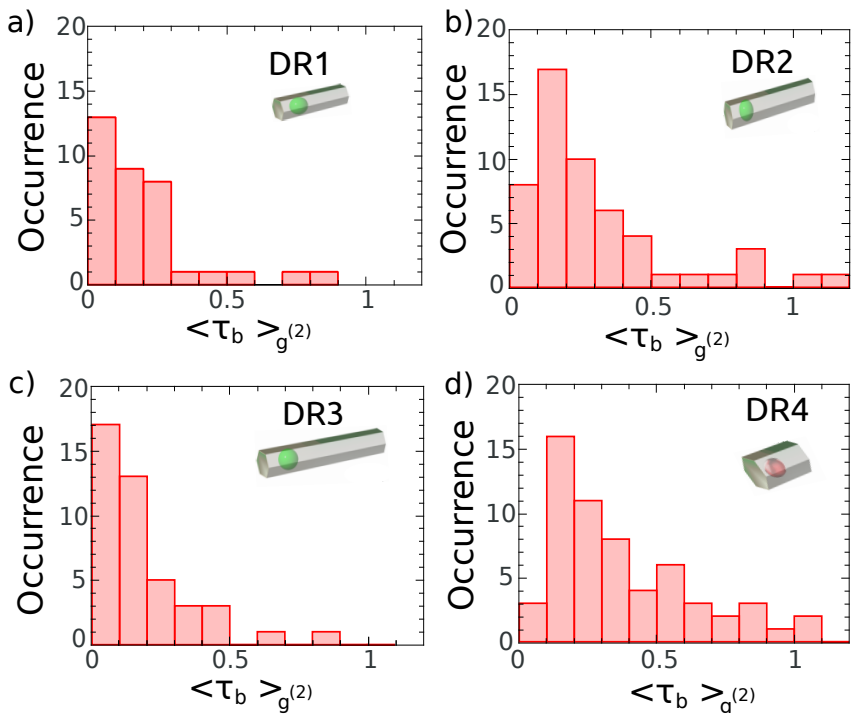

FIG. 5. Histogram of the average bright period duration for each studied sample. The samples mean values of $\left\langle\tau_{b}\right\rangle_{g(2)}$ are: a) DR1: $190 \mu \mathrm{s}(\sigma=189 \mu \mathrm{s})$, b) DR2: $299 \mu \mathrm{s}(\sigma=264 \mu \mathrm{s})$, c) DR3: $186 \mu \mathrm{s}(\sigma=175 \mu \mathrm{s})$, d) DR4: $394 \mu \mathrm{s}(\sigma=299 \mu \mathrm{s})$.

fast. We define the minimum duration of a bright or grey event $\tau_{\min }$ in eq 4 as the delay $\Delta$ between two excitation pulse as proposed in reference ${ }^{32}$, here $\tau_{\min }=400 \mathrm{~ns}$. This is reasonable as long as the excitation repetition rate is low enough. Indeed, in this case the resulting delay between pulses is larger than the typical timescale of any physical mechanism that would prevent the switching between states. Therefore in this case $\tau_{\min }$ is fixed by the excitation rather than by a physical mechanism.

The rate of excitation of a nanocrystal can heavily modify the blinking statistics, and thus the cumulative distribution of events $\mathcal{P}\left(\tau_{b, g} \geq \tau\right)$ and the corresponding $g^{(2)}$ function, as shown in reference ${ }^{38}$. Hence we do not consider a change of the rate of excitation experimentally because this might lead to compare blinking statistics for a given nanocrystal with different power law exponents $\mu$ for example. In this case one would not be able to sort out the effect of changing the minimum delay between single photons $\tau_{\min }$ on the average blinking duration with any other potential changes in the blinking statistics. To test the effect of the repetition rate on the 

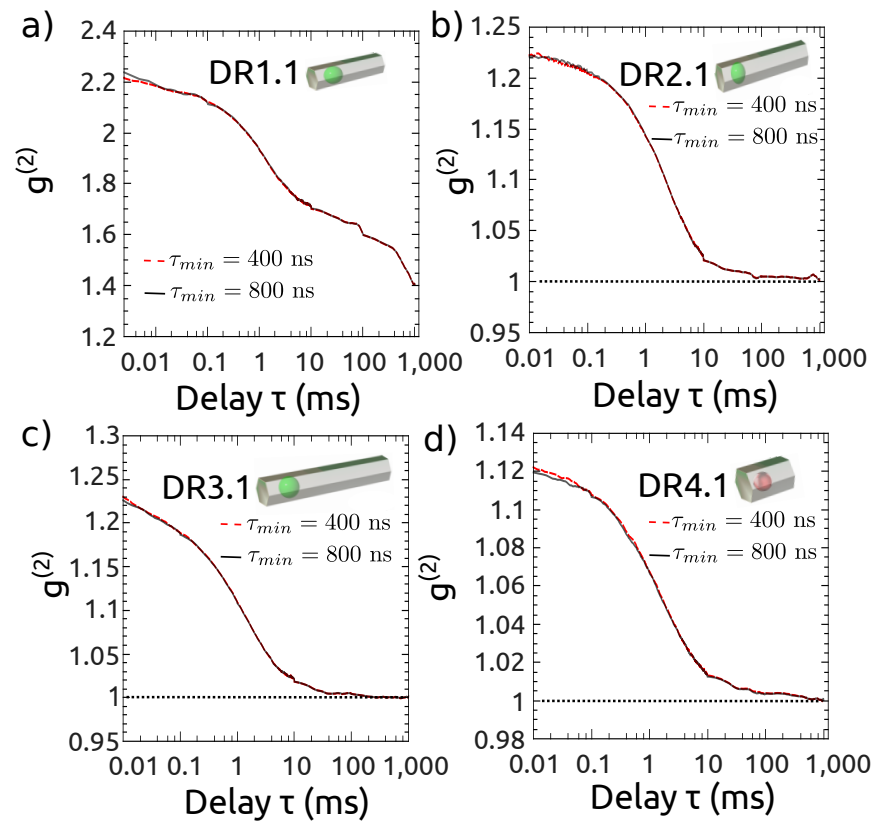

FIG. 6. $\quad g^{(2)}$ function for DR1.1, DR2.1, DR3.1 and DR4.1 from samples DR1, DR2, DR3 and DR4 respectively. The red dashed curve is the $g^{(2)}$ function calculated from the raw data as already presented in fig 2 and fig 3 with $\tau_{\min }=400 \mathrm{~ns}$. The solid black line is the $g^{(2)}$ function calculated from the raw data keeping only every second excitation such that $\tau_{\min }=$ 800 ns. $\left\langle\tau_{b}\right\rangle_{g^{(2)}}$ values via the $\tau_{\min }$ parameter in eq, 4 , we decrease the repetition rate by removing detected photons in the post-measurement data analysis. By removing every second excitation from a measurement, we can artificially simulate a decrease by a factor of 2 of the repetition rate.

In fig 6 we present the $g^{(2)}$ function of the nanocrystals studied in fig 2 and fig 3 calculated on all the photons registered experimentally (red dashed curve), and on only every second excitation (solid black line). The $g^{(2)}$ functions are almost identical. This implies that the fits give $\left\langle\tau_{b}\right\rangle_{g^{(2)}, \tau_{\min }=800 \mathrm{~ns}} \simeq\left\langle\tau_{b}\right\rangle_{g^{(2)}, \tau_{\min }=400 \mathrm{~ns}} \times 2^{\mu}$ according to equ The average bright period duration increases while increasing the minimum blinking duration $\tau_{\min }$ as fast blinking events are removed from the statistics. Table III gives the average blinking duration found trough the fitting procedure for the four nanocrystals under study for both $\tau_{\min }=400 \mathrm{~ns}$ and $\tau_{\min }=800 \mathrm{~ns}$.
1 M. Nirmal, B. Dabbousi, M. Bawendi, J. Macklin, J. Trautman, T. Harris, and L. Brus, Nature 383, 802 (1996).

2 Geng Wei, Manceau Mathieu, Rahbany Nancy, Sallet Vincent, De Vittorio Massimo, Carbone Luigi, Glorieux Quentin, Bramati Alberto, and Couteau Christophe, Scientific Reports 6, 19721 (2016)

3 B. Lounis and M. Orrit, Reports on Progress in Physics 68, 1129 (2005).

4 O. A. Shcherbina, G. A. Shcherbina, M. Manceau, S. Vezzoli, L. Carbone, M. D. Vittorio, A. Bramati, E. Giacobino, M. V. Chekhova, and G. Leuchs, Opt. Lett. 39, 1791 (2014)

5 P. A. Frantsuzov, S. Volkán-Kacsó, and B. Jankó, Nano letters 13, 402 (2013).

${ }^{6}$ C. Bradac, T. Gaebel, N. Naidoo, M. Sellars, J. Twamley, L. Brown, A. Barnard, T. Plakhotnik, A. Zvyagin, and J. Rabeau, Nature nanotechnology 5, 345 (2010).

7 M. Kuno, D. Fromm, H. Hamann, A. Gallagher, and D. Nesbitt, The journal of chemical physics 112, 3117 (2000).

8 X. Brokmann, J.-P. Hermier, G. Messin, P. Desbiolles, J.P. Bouchaud, and M. Dahan, Physical review letters 90, 120601 (2003).

9 B. Mahler, P. Spinicelli, S. Buil, X. Quelin, J.-P. Hermier, and B. Dubertret, Nature materials 7, 659 (2008).

10 Y. Chen, J. Vela, H. Htoon, J. L. Casson, D. J. Werder, D. A. Bussian, V. I. Klimov, and J. A. Hollingsworth, Journal of the American Chemical Society 130, 5026 (2008).

\begin{tabular}{|l|c|c|}
\hline & $\begin{array}{c}\left\langle\tau_{b}\right\rangle_{g^{(2)}} \\
\tau_{\min }=400 \mathrm{~ns}\end{array}$ & $\begin{array}{c}\left\langle\tau_{b}\right\rangle_{g^{(2)}} \\
\tau_{\min }=800 \mathrm{~ns}\end{array}$ \\
\hline \hline DR1.1 & $150 \mu \mathrm{s}(12)$ & $213 \mu \mathrm{s}(23)$ \\
\hline DR2.1 & $1250 \mu \mathrm{s}(309)$ & $1595 \mu \mathrm{s}(478)$ \\
\hline DR3.1 & $42 \mu \mathrm{s}(8)$ & $86 \mu \mathrm{s}(15)$ \\
\hline DR4.1 & $465 \mu \mathrm{s}(122)$ & $698 \mu \mathrm{s}(211)$ \\
\hline
\end{tabular}

TABLE II. Average bright period duration $\left\langle\tau_{b}\right\rangle_{g^{(2)}}$ and its uncertainty found from a fit of the correlation function for DR1.1, DR2.1, DR3.1 and DR4.1 for $\tau_{\min }=400 \mathrm{~ns}$ and $\tau_{\text {min }}=800 \mathrm{~ns} . \tau_{\text {min }}=400 \mathrm{~ns}$ corresponds to the raw data as presented in fig 2 and fig 3 while $\tau_{\min }=800 \mathrm{~ns}$ corresponds the the same data for which every second excitation was removed.

11 P. Spinicelli, S. Buil, X. Quelin, B. Mahler, B. Dubertret, and J.-P. Hermier, Physical review letters 102, 136801 (2009).

12 A. V. Malko, Y.-S. Park, S. Sampat, C. Galland, J. Vela, Y. Chen, J. A. Hollingsworth, V. I. Klimov, and H. Htoon, Nano letters 11, 5213 (2011).

13 C. Galland, Y. Ghosh, A. Steinbrück, M. Sykora, J. A. Hollingsworth, V. I. Klimov, and H. Htoon, Nature 479, 203 (2011).

14 C. Galland, Y. Ghosh, A. Steinbrück, J. A. Hollingsworth, H. Htoon, and V. I. Klimov, Nature communications 3, 908 (2012). 
15 D. V. Talapin, R. Koeppe, S. Götzinger, A. Kornowski, J. M. Lupton, A. L. Rogach, O. Benson, J. Feldmann, and H. Weller, Nano Letters 3, 1677 (2003).

16 D. V. Talapin, J. H. Nelson, E. V. Shevchenko, S. Aloni, B. Sadtler, and A. P. Alivisatos, Nano letters 7, 2951 (2007).

17 L. Carbone, C. Nobile, M. De Giorgi, F. D. Sala, G. Morello, P. Pompa, M. Hytch, E. Snoeck, A. Fiore, I. R. Franchini, et al., Nano letters 7, 2942 (2007).

18 S. Ithurria, G. Bousquet, and B. Dubertret, Journal of the American Chemical Society 133, 3070 (2011).

19 Y. Louyer, L. Biadala, J.-B. Trebbia, M. Fernée, P. Tamarat, and B. Lounis, Nano letters 11, 4370 (2011).

20 M. D. Tessier, C. Javaux, I. Maksimovic, V. Loriette, and B. Dubertret, ACS nano 6, 6751 (2012).

${ }^{21}$ L. Biadala, B. Siebers, R. Gomes, Z. Hens, D. R. Yakovlev, and M. Bayer, The Journal of Physical Chemistry C 118, 22309 (2014).

22 S. Vezzoli, M. Manceau, G. Leménager, Q. Glorieux, E. Giacobino, L. Carbone, M. De Vittorio, and A. Bramati, ACS nano 9, 7992 (2015).

${ }^{23}$ F. Pisanello, G. Leménager, L. Martiradonna, L. Carbone, S. Vezzoli, P. Desfonds, P. D. Cozzoli, J.-P. Hermier, E. Giacobino, R. Cingolani, et al., Advanced Materials 25, 1973 (2013).

24 S. Vezzoli, S. Shojaii, S. Cialdi, D. Cipriani, F. Castelli, M. G. Paris, L. Carbone, P. D. Cozzoli, E. Giacobino, and A. Bramati, Optics Communications 300, 215 (2013).

25 M. Manceau, S. Vezzoli, Q. Glorieux, F. Pisanello, E. Giacobino, L. Carbone, M. De Vittorio, and A. Bramati, Physical Review B 90, 035311 (2014).

26 C. H. Crouch, O. Sauter, X. Wu, R. Purcell, C. Querner,
M. Drndic, and M. Pelton, Nano letters 10, 1692 (2010).

27 N. Amecke, H. Heber, F. Cichos, The Journal of chemical physics 140, 114306 (2014).

28 J. Bernard, L. Fleury, H. Talon, and M. Orrit, The Journal of chemical physics 98, 850 (1993).

29 L. Fleury, J.-M. Segura, G. Zumofen, B. Hecht, and U. Wild, Physical review letters 84, 1148 (2000).

30 G. Messin, J. Hermier, E. Giacobino, P. Desbiolles, and M. Dahan, Optics Letters 26, 1891 (2001).

31 R. Verberk, A. M. van Oijen, and M. Orrit, Physical Review B 66, 233202 (2002).

32 M. Pelton, D. G. Grier, and P. Guyot-Sionnest, Applied physics letters 85, 819 (2004).

33 M. Pelton, G. Smith, N. F. Scherer, and R. A. Marcus, Proceedings of the National Academy of Sciences 104, 14249 (2007).

34 D. Canneson, L. Biadala, S. Buil, X. Quélin, C. Javaux, B. Dubertret, and J.-P. Hermier, Physical Review B 89, 035303 (2014).

35 J. Houel, Q. T. Doan, T. Cajgfinger, G. Ledoux, D. Amans, A. Aubret, A. Dominjon, S. Ferriol, R. Barbier, M. Nasilowski, et al., ACS nano 9, 886 (2015).

36 R. Verberk and M. Orrit, The Journal of chemical physics 119, 2214 (2003).

37 I. Sychugov, R. Juhasz, J. Linnros, J. Valenta, Physical Review B 71, 115331 (2005).

38 O. Schwartz, R. Tenne, J. M. Levitt, Z. Deutsch, S. Itzhakov, and D. Oron, ACS nano 6, 8778 (2012).

39 M.E. Pistol, P. Castrillo, D. Hessman, J.A. Prieto, and L. Samuelson, Physical Review B 59, 10725 (1999). 